\title{
The Impact of Marketing Ethics on Customer Loyalty: A Conceptual Framework
}

\section{Rami M. M. Nassar}

University Sians Islam Malaysia (USIM)

Nilai, Malaysia

Email: $\underline{\text { rami_118@hotmail.com }}$

\section{Mohamed Battour}

University of Sharjah

Sharjah, United Arab Emirates

Email: mbattour@usim.edu.my

Received March, 2020; Accepted May, 2020

\begin{abstract}
The purpose of this study is to develop a conceptual framework to identify the impact of marketing ethics on customer loyalty in Palestine. The study relies on two mobile phone companies: Jawwal and Ooredoo Palestine. It accounts for the Palestinian environment, which is characterized by political and economic instability, due to the Israeli occupation since 1948, the division of the homeland (the West Bank and the Gaza Strip), and the Israeli siege on the Gaza Strip. The study contributes to pinpoint strengths and weaknesses of both companies and shed light on their marketing and ethical behaviors. Findings show that customer satisfaction greatly affects marketing strategies and ethical behavior. The study concludes to important remarks. Palestinian companies are encouraged to develop their marketing plans and highlight surrounding ethical standards to address special characteristics of the Palestinian society. This will increase customer satisfaction, retention, and loyalty which will positively affect company's profitability and performance.
\end{abstract}

Keywords: Marketing Ethics; Customer Loyalty; Cellular Telecomunications Companies; Palestine.

Type: Research paper

الغرض من هذه الدراســـة هو تطوير إطار مفاهيمي لتحديد تأثير أخلاقيات التســويق على ولاء العملاء من خلال مراجعة الأدبيات السابقة الأكثر صلة بهذا الموضوع. علاوة على ذلك، إلقاء الضوء على واقع أخلاقيات التسويق في شـــركتي الهاتف المحمول العاملتين في فلســـطين، وهما جوال وأوريدو فلســــين، من خلال التركيز على البيئة الفلسـينية، التي تتميز بعدم الاسـتقرار السـياسـي والاقتصـادي، بسـبب الاحتلال الإسـرائيلي لفلسـين منذ 1948 وكذلك الانقسـام الفلسـيني بين شطري الوطن (الضـفة الغربية وقطاع غزة) والحصـار الإسـرائيلي على قطاع غزة. ومن ثم، فمن المتوقع أن تشكل هذه الدراسـة مرجعا قيما لهاتين الثـركتين لتطوير سلوكياتهما التسويقية والأخلاقية. وبناءً عليه، يتم التحقيق في أثر رضـا العملاء على الأداء التسـويقي والأخلاقي لهاتين الثـركتين والخدمات المقدمة 
من قبلهما من أجل تطوير خططهما التســويقية وتطوير معايير أخلاقية متوافقة مع المجتمع الفلــــيني، ومن ثم زيادة أرباح الثركة التي يمكن تحقيقه من خلال رضا العملاء عن خدماتهم وكسب ولائهم.

الكلمات المفتاحية: أخلاقيات التسويق، ولاء الزبون، شركات الاتصالات الخلوية، فلسطين.

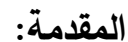

تسعى العديد من الثركات إلى زيادة حصتها السوقية وجني مزيد من الأبباح، ويرتكز نجاح الإدارة التسويقية على مصداقية الأفراد ذوي العلاقة بالتحليل والتنفيذ وعلى خطة العمل، فإن اعتمدت إدارة التسويق في أي منظمة على طرق من الخداع والتضليل في تسويق منتجاتها، وابتعد موظفوا هذه الإدارة عن أخلاقيات التسويق، وكان الهم الأوحد لهح هو جني الأرباح دون الاهتمام برضا الزبون وسد احتياجاته، لن تحافظ المنظمة على زبائنها، ولن يكون هناك نمو أو ديمومة لمبيعات هذه المنظمة وولاء الزبون لها (كافي، 2009). ورغم ذلك ففي مجتمعاتتا العربية والاسلامية ظهرت العديد من الثركات والمؤسسات التي تستخدم الدعاية المضللة والإعلانات الكاذبة أو ما يعرف بالخداع التسويقي، بعيداً عن أخلاقيات المجتمعات الاسلامية وعاداتهم، بهدف جني مزيد من الأرباح بالغش والخداع والتضليل، وتكون الخدمات المقدمة من قبل هذه الشركات ظاهرها يختلف عن حقيقتها دون أي مسؤولية اجتماعية أو دينية تجاه الفرد أو المجتمع، مع أن الاسلام حث على الايفاء بالعهد وعدم الغش فالهه جل وعلا قال في كتابه العزيز : "وأوفوا بالعهد إن العهد كان مسؤولاً" (الاسراء :34).

فلسطين هي جزء لا يتجزأ من الوطن العربي والبيئة العالمية، تتأثر بكل ما هو جديد من الأساليب والأدوات التسويقية والترويجية المستحدثة في مختلف القطاعات سواء كانت صناعية أوخدماتية، وقد يكون أن بعض الثركات العاملة فيها أيضاً لا تلتزم بشكل كبير بالأخلاقيات التسويقية والدعاية السليمة البعيدة عن الغش والخداع، وقطاع الاتصالات الفلسطيني سواء الأرضي أو الخلوي من أهم القطاعات العاملة في فلسطين، ويعمل في هذا القطاع شركتى جوال وأوريدو فلسطين، لذلك يتوجب على هاتين الثركتين الالتزام بأخلاقيات التسويق وجودة الخدمات، وألا تستغل حاجة المواطن الفلسطيني للخدمات المقدمة من قبلها عبر اعلانات تسويقية براقة، ولكنها خالية من المضمون والجودة، مما سيعود بالضرر أولاً على مبيعات وسمعة هذه الشركات وعلى ولاء الزبائن لها، وبالتالي سيعود بالضرر على الفرد والمجتمع في قطاع غزة.

ولقد كان لاخول شركة أوريدو فلسطين سوق العمل الخلوي في قطاع غزة عام 2017م تأثير تتافسي تسويقي كبير بين هاتين الثركتين، من جهة شركة جوال وجدت نفسها تعمل في بيئة فيها منافس حقيقي لم تتعود عليه منذ ثمانية عشرة عاماً في قطاع غزة، وكذلك أيضاً انطلاق أعمال شركة جديدة كشركة أوريدو فلسطين في قطاع غزة تعتبر منافس قوي لشركة جوال، أدى بالشركتين من اطلاق حملات تسويقية كبيرة، رغبة من شركة جوال المحافظة على زبائنها وكسب ولائهم، وكذلك زيادة مبيعاتها في خضم هذه الأجواء الجديدة من المنافسة الحقيقية، وعلى الطرف الآخر شركة أوريدو فلسطين والتي تعتبر سوق ناشئ في قطاع غزة، يحتاج لاخول السوق بقوة والحصول على رضا 
الجمهور من خلال أعمال تسويقية تجذبه إليها، وخدمة حقيقية ذات جودة عالية في ظل منافس قوي، والذي كان محتكراً لهذا المجال في قطاع غزة لفترة طويلة من الزمن.

وقد أكدت العديد من الدراسات أن هناك علاقة متينة بين أخلاقيات التسويق وولاء الزبون من جهة، وكذلك جودة الخدمة وولاء الزبون من جهة أخرى، فقد أظهرت دراسة بن جروة وبن حوحو بوجود علاقة ذات دلالة إحصائية بين الالتزام بأخلاقيات التسويق والسلوك الشرائي لزبائن المحلات التجارية لعينة الدراسة، بمعنى أن لأخلاقيات التسويق ارتباط بولاء الزبون (بن جروة، بن حوحو، 2017)، بينما أظهرت بعض الدراسات غير ذلك، فقد أظهرت دراسة (الدويك، 2006) من خلال مؤشر الولاء بأن ثلث المشتركين في شركة جوال يفكرون في الانتقال من الشركة، وأنه في حالة وجود شركة فلسطينية بديلة ربما ستتضاعف هذه النسبة، وكذلك أظهرت أن العلاقة بين المواطنين والثركة جاءت بنسبة متوسطة، وان هناك نسبة غيرراضية عن مدى اهتمام الشركة بالمواطنين.

وبعد مراجعة العديد من الدراسات حول أخلاقيات التسويق وولاء الزبون، وجد ندرة في الأبحاث التي تم إجراءها على البيئة العربية ومنها الفلسطينية، فقد ظهر أن منظمات الأعمال العربية لم تعطي هذا المجال الاهتمام الكافي، حيث تبين أن الكثير من الدراسات العربية في هذا الموضوع جاءت متأثرة بما قدمه الباحثين الغربيين من أفكار، وقد لا لاعله تتاسب هذه الأفكار واقعنا العربي والاسلامي (الهنداوي، واخرون، 2010)، لذلك تهدف هذه الدراسة الى تسليط الضوء على مفاهيم أخلاقيات التسويق من خلال مراجعة الدراسات السابقة وكذلك علاقة تلك الأخلاقيات بولاء الزبون وواقعها في شركتي جوال وأوريدو فلسطين، وعليه فإنه من المتوقع أن تثكل هذه الدراسة مرجع حقيقي للشركتين محل الدراسة لتطوير عملها التسويقي، ومعرفة مدى رضا الجمهور عن أدائهما التسويقي وعن الخدمات المقدمة من قبل هاتين الثركتين، من أجل تطوير خططهما التسويقية ووضع معايير أخلاقية متوافقة والمجتمع الفلسطيني، وتطبيق هذه المعايير على أرض الوقع، مما يعود بالنفع على الشركتين وعلى الخدمات المقدمة للجمهور الفلسطيني، من خلال التركيز على البيئة الفلسطينية التي تتميز بعدم الاستقرار السياسي والاقتصادي على مدار مدة طويلة من الزمن وحتى الآن بسبب الاحتلال الاسرائيلي لفلسطين منذ 1948م. وعليه فان هذه الدراسة سوف يتم تقسم هيكلها كما

القسم الثاني يتتاول واقع الأتصالات الخلوية في فلسطين، أما القسم الثالث فسيتم تسليط الضوء على الإطار المفاهيمي و الدراسات السابقة المرتبطة بموضوع الدراسة، فيما سيتضمن القسم الرابع منهجية الدراسة.

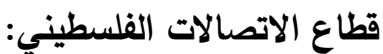

كان قطاع الاتصالات في فلسطين في بداياته ملكاً للسلطة الوطنية الفلسطينية، لكن بعد ذلك و بموجب قرار السلطة الذي أصدر بتاريخ 15 تشرين ثاني 1996م، أصبح هذا القطاع من صلاحيات شركة الاتصالات الفلسطينية، حيث أنشئت هذه الثركة بموجب اتفاقية بهذا الخصوص وقعت مع السلطة الوطنية الفلسطينية الممثلة بوزارة البريد والاتصالات، هذه الاتفاقية أعطت الثركة صلاحية إنشاء وتركيب وتثغيل كل من الهاتف الثابت والخلوي، وكل ما 
يتعقق بهما من تجهيزات فنية....، ويسري مفعول هذه الرخصة لمدة عشرين عاماً من تاريخ التوقيع قابلة للتجديد، وبذلك أصبحت شركة الاتصالات الفلسطينية المساهمة العامة المحدودة (PALTEL) الشركة الفلسطينية الوحيدة المشغلة للاتصالات الثابتة والخلوية في فلسطين وبرأسمال ابتدائي 45 مليون دينار أردني. (بالتل، التقرير السنوي:

\section{1}

تعتبر شركة الاتصالات الخلوية الفلسطينية (جوال) أول شركة اتصالات فلسطينية متخصصة في الاتصالات

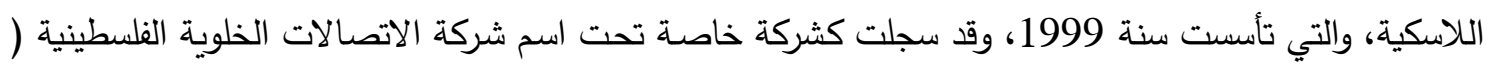
في أيلول سنة 2000)، وعليه فقد تم الإعلان عن استقلاليتها العملية والوظيفية والمالية عن شركة الاتصالات الفلسطينية (بالتل) في كانون الثاني 2000، و قد بدأت شركة الاتصالات الخلوية الفلسطينية (جوال) برأس مال مصرح ومكتتب بـه بقيمة خمسة وعشرون مليون دينار أردني.

حيث كانت تساهم فيها كل من شركة الاتصالات الفلسطينية وشركة الخدمات التجارية التي تمثل السلطة الوطنية الفلسطينية، بملكية 65\% و35\% على التوالي، وفيما بعد قامت شركة الاتصالات الفلسطينية بشراء كامل مساهمة شركة الخدمات التجارية في شركة جوال، لتصبح الشركة مملوكة بالكامل لشركة الاتصالات الفلسطينية. (عبد العال، .$(2007$

\section{2. شركة أوريدو فلسطين:}

قامت الثركة بتاريخ 14 آذار 2007 بتوقيع اتفاقية مع وزارة الاتصالات وتكنولوجيا المعلومات الفلسطينية، منحت من خلالها الثركة رخصة لتثغيل” خدمات الجيل الثاني والثالث في الضفة الغربية وقطاع غزة، مدة الرخصة عشرين سنة تبدأ من 10 أيلول 2009 ، وهذا التاريخ يمثل فعلياً حصول الشركة على ترددات خدمة الجيل الثاني في الضفة الغربية.

وقد أطلقت شركة أوريدو الفلسينية خدماتها التجارية في شهر تشرين الثاني من العام 2009، ربدات عملها فقط في الضفة الغربية نظراً لمنع الاحتلال لها من العمل في قطاع غزة، ولكن شهد العام 2017 انطلاق لعمل هذه الشركة في قطاع غزة، ليشهد قطاع الاتصالات الفلسطينية الخلوية أجواء من المنافسة بعدما كان هذا القطاع في قطاع غزة حكراً على شركة (جوال) فقط (أوريدو فلسطين، اكتتاب ثانوي عام: 2018).

الاطار المفاهيمي و الاراسات السابقة: - المات

يتضمن هذا القسم الاطارالنظري والدراسات السابقة المتعلقة بأخلاقيات التسويق، و ولاء الزبون، وتم تقسيم الاطار النظري إلى ثلاث أقسام رئيسية: القسم الاول يقدم مفهوم أخلاقيات التسويق وأبعاده، والقسم الثاني يتحدث عن ولاء 
الزبون من حيث المفهوم والأهمية، بينما يحتوي القسم الثالث على الاحتلال والانقسام الفلسطيني وآثارهما الاقتصادية، فيما القسم الرابع يتكون من الدراسات السابقة.

\section{اولاً: مفهوم أخلاقيات التسويق:}

تعتبر الأخلاقيات موضوع حيوي يحتل أهمية كبيرة و ذلك بسبب زيادة حاجة المؤسسات إليها وضرورتها في عالمنا اليوم، بعد أن أصبحت المعايير المادية وزيادة أرباح المؤسسات بشتى الوسائل هي التي تحكم العلاقات بين البائع والزبون أكثر من الدين والقيم والأعراف و التقاليد. تعددت مفاهيم الأخلاق ولكنها في معظمها لا تختلف كثيرا من حيث المضمون والجوهر، لكن ربما الاختلاف فقط من حيث الأسلوب والثكل، وهنا نستعرض بعض من هذه التعريفات:

فقد عرفها نجم (2006) و المرهضي (2009) بأنها "عبارة عن مفهوم المجتمع لما هو صواب أو خطأ في السلوك". بينما عرفها الطراونة وأبو جليل بأنها: مجموعة من القواعد والمبادئ والأحكام السلوكية والأدبية والقيم والمعايير والمقاييس الأخلاقية التي تحكم تصرفات الأفراد والجماعات أثثاء تأدية الأعمال في بيئة العمل، وتثمل هذه الخصائص الموضوعية والاستقلالية وكذلك الأمانة والاستقامة والنزاهة والثفافية، والإعتمادية والمسؤولية. (الطراونة وآخرون، 2013)

ثانياً: أبعاد أخلاقيات التسويق:

هناك العديد من الأبعاد والعناصر الأساسية لأخلاقيات التسويق التي لا بد من توفرها في العاملين والموظفين في أي منظمة أعمال وسيتم التركيز في هذه الدراسة على البعدين التاليين:

1- المواطنة: والتي تتحقق باحترام المنظمات والمؤسسات للأنظمة والقوانين، والتي لا بد من تحقيقها من أجل الامتثال للقوانين والأنظمة الوطنية. (يوسف، 2015).

2- الاحترام: أي احترام قيم وعادات المجتمع أثناء الاعلانات وخلال عملية البيع والثراء والتفاوض والاتصالات والعلاقات، حيث أكدت النظريات الحديثة على أهمية الاحترام وجعلته من أهم استراتيجيات المؤسسات

$$
\text { الناجحة. (العمر، 1999). }
$$

ثالثاً: مفهوم ولاء الزبون:

تعددت مفاهيم ولاء الزبون، وتداول الباحثون عديد المفاهيم بهذا الخصوص، فالولاء هو عبارة عن مزيج من المعاني النفسية والعاطفية كالإيمان بأمر معين أو الميل نحوه، ولكن السلوك الفعلي للزبون وليست العواطف وحدها هو من يحقق الربحية المرجوة. فقد وضح (day,1969) و (Chen, et, al., 2009) مفهوم ولاء الزبون بأنه الاستجابة السلوكية المنحازة كلياً لشركة أو منتج ما بثكل مستمر ودائم بالرغم من وجود بديل أو أكثر يحمل نفس المزايا وربما 
أكثر جودة. وقد عرفه البعض بأنه عبارة عن قيام المنظمات أو الشركات باستخدام حواجز أو موانع طبيعية تمنع تحول الزبون إلى منافس آخر (توفيق، 2007). كما عرفه أيضاً (Fournier\& yao, 1997: 452) بأنه الالتزام بإعادة الثراء أو إعادة اقتتاء المنتج بثكل مستمر ودائم في المستقبل بالرغم من تأثير الجهود التسويقية الكبيرة

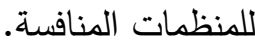

$$
\text { رابعاً: أهمية ولاء الزبون: }
$$

تتلخص أهمية ولاء الزبون لأي منظمة أو مؤسسة من خلال النقاط التالية: 1- 80\% من عملك يأتي من 20\% من الزبائن: قاعدة 20180 تظهر أن معظم الشركات وجدت أن 80\% من منتجاتها يشتريها 20\% فقط من زبائنها، وهذا يوجب على هذه الثركات المحافظة على هذه الفئة لكسب ولائهم

ورضاهم لاستمرار زيادة المبيعات ونمو الأرباح لديهم (Furlong, 1993; Alareeni, 2018; 2019). 2- الكلمة المنطوقة: هي أرخص شكل من أشكال التسويق، بحيث الزبون الراضي عن تعامل الشركة وعن منتجاتها بالتأكيد سيصبح مدير تسويق مجاني للشركة، عبر الحديث لأصدقائه وأقربائه عن جودة هذا المنتج وحسن معاملة الشركة، وبالتالي سيؤدي ذلك لكسب زبائن جدد للشركة، و من فوائد الكلمة المنطوقة الإيجابية، خاصة التي تصدر من العملاء ذوي الرضا العالي، أنهم يعتبرون بمثابة إعلان متحرك للشركة (سويدان،2011). 3- التكلفة: أظهرت الدراسات الإحصائية التي نشرتها مجلة الاتصالات و العالم الرقمي بتاريخ 2006/1/22، أن "معدل فقدان الزبائن لاى الثركات قد يبلغ 20\% من عدد العملاء الكلي سنوياً، وأن كلفة عملية المحافظة على العميل قد تبلغ ست أضعاف كلفة اكتساب عميل جديد، وأن 68\% من العملاء يغيرون الشركات والمؤسسات التي يتعاملون معها بسبب الخدمات، والملاحظ أن 4\% فقط من هؤلاء العملاء الذين توقفوا عن التعامل سبق لهم أن اشتكوا من سوء الخدمات، بينما 90\% تركوا الثركات دون سابق إشعار، وأن 82\%من العملاء الذين تم حل مشكلاتهم عاودوا التعامل مع الشركات نفسها”.( كامل، 2006).

خامساً: الاحتلال والانقسام وآثارهما الاقتصادية:

تعرضت مؤسسات السلطة الفلسطينية في 2007/6/14 لانقسام سياسي حاد، هذا الانقسام كانت له توابع عديدة على كافة الصعد، اقتصادي واجتماعي وسياسي، ومنها ما هو داخلي ومنها ما هو وخارجي، وقطاع الاتصال الفلسطيني بما فيه الخلوي تأثر بهذه التوابع بشكل سيئ. والحالة الاقتصادية في أي بلد تتأثر بالعوامل الخارجية والبيئة الجغرافية، وأبرز ما تواجهه الحالة الاقتصادية في فلسطين وخاصة قطاع غزة هو الاحتلال والانقسام الفلسطيني، فالاحتلال وحصاره بالاضافة للانقسام أثروا بشكل سلبي على النمو الاقتصادي وتطوره، لصعوبة التواصل الجغرافي بين الضفة وقطاع غزة، والقيود التي يفرضها الاحتلال على الترابط الاقتصادي بين شطري الوطن، وكذلك الحروب المتكررة على قطاع غزة، بالاضافة لغياب خطة تتموية شاملة( أبو حماد، 2011) .إن الدول التي تعصف بها الحروب والنزاعات الداخلية لا تثهد مقتل أعداد كبيرة من سكانها فقط، بل تتأثر مواردها الطبيعية بشكل كبير وسلبي، 
Shamma,1986;Alareeni and ) ويؤدي إلى تقويض مؤسساتها الوطنية، وتعطيل خطط التتمية والتقدم فيها Branson, 2013 . وقد ترك الانقسام الفلسطيني والاحتلال آثاره بشكل كبيرعلى شتى مجالات الحياة السياسية والاجتماعية والاقتصادية والنفسية كافة في قطاع غزة، ففي الجانب الاقتصادي تقلصت كمية ومستوى الانتاج، وتحول المجتمع واقتصاده إلى اقتصاد إغاثي أكثر من كونه اقتصاد منتج ( مرتجى، 2014).

أظهرت دراسة بودرجة وصليحة بأن الشركات الصناعية الغدائية لعينة الدراسة تلتزم بأخلاقيات التسويق، ولهذا الأمر أثر معنوي على صورتها الذهنية المدركة لدى الزبون (بودرجة، صليحة، 2017). وكذلك أوضحت دراسة بن جروة وبن حوحو بوجود علاقة ذات دلالة إحصائية بين الالتزام بأخلاقيات التسويق والسلوك الشرائي لزبائن الدحلات التجارية لعينة الدراسة، بمعنى أن لأخلاقيات التسويق ارتباط بولاء الزبون (بن جروة، بن حوحو، 2017).. بينما أظهرت دراسة كحلي بأن ممارسة المؤسسات للمسؤولية الاجتماعية والأخلاقية يحسن من صورتها وسمتتها لدى المستهلك وبالتالي يقوي من ولاء الزبون لها ولمنتجاتها، وكذلك يساهم الزبون بشكل إيجابي في التأثير على المؤسسات لدفعها نحو تبني الأخلاقيات التسويقية والمسؤولية الاجتماعية، فيما أظهرت نتائج العينة المستهدفة أن أخلاقيات التسويق تؤثر على لئى سلوكهم الاستهلاكي (كحلي، 2016). بينما خلصت دراسة بن صالح بأن التسويق في مجمله يهدف إلى التأثير على سلوك المستهلك، وأن الأخلاق لها ارتباط كبير في التسويق، ما يتجلى في كثرة القضايا والمسائل التي ترتبط مضمونا ومنهجاً بالأخلاق سواء من حيث مبادئ التسويق و أصوله، أو من حيث أهدافه ومضامينه كما بينت نتائج الدراسة الميدانية وجود أثر ذو دلالة إحصائية للأخلاق على سلوك المستهلك قبل أو بعد عملية الشراء، ويتغيرذلك الأثر بتغير مستوى إدرالك المستهلك لأهمية أخلاقيات التسويق (بن صالح، 2014).

كما وضحت دراسة خدير (2011) بأن توفر السلوك الأخلاقي في المؤسة يوطد العلاقة بين المؤسسة والعمال وكذلك مع مختلف أصحاب المصالح وخصوصا الزبائن، كما أن توفر سلوكيات الأخلاق في أي مؤسسة يؤدي إلى ئى الثفافية، وبالتالي يحقق ذلك رضا الموظفين والزبائن. وكذلك بينت دراسة حجاوي أن صدق الأسعار المثبتة على المنتجات تؤثر على نظرة المستهلك الفلسطيني للأبعاد الأخلاقية للمسؤولية الاجتماعية، وأن صدق الرسائل الترويجية تؤثر بنسبة كبيرة على نظرة المستهلك الفلسطيني للأبعاد الأخلاقية كما يدركها المستهلك( حجاوي، 2011). بينما وضحت دراسة جويل أن رضا الجمهور عن الخدمات المقدمة لا يعني زيادة في ولاء الزبون، فقد أظهرت هذه الدراسة أن ما يقرب من (65\%-85\%) من المسافرين الذين قاموا بتغيير شركة الطيران التي كانوا يتعاملون معها كان لديهم الرضا والرضا الثديد عن خدمات الثركات التي كاناو يتعاملوان معها إلا أن نسبة ما يقرب من (30\%-40\%) هم من عاودوا التعامل مع نفس الثركة مرة، وهذا يعني أن الرضا عن الخدمات المقدمة لا يعني زيادة الولاء (جويل، 2001). فيما أظهرت دراسة فلوب وهسريتش وسجيدي إلى أن ما نسبته 22\% من المنظمات الهنغارية لا تهتم بالقضايا الأخلاقية، و أشار أفراد العينة إلى أن عدم الاهتمام بأخلاقيات الأعمال ناتج عن عدة أسباب أهمها النقص 
المعرفي في هذا المجال، وأن أغلب المنظمات تهتم بالبقاء أكثر من الاهتمام بقاضايا الأخلاق \& Fulop). Hisrich \& Szegedi, 2000)

\section{منهجية الدراسة:}

تعتبر منهجية الدراسة وإجراءاتها محوراً رئيساً، من خلالها يتم الحصول على البيانات المطلوبة للتوصل إلى النتائج التي يتم تفسيرها في ضوء أدبيات الدراسة المتعلقة بموضوع الدراسة، وبالتالي تحقق الأهداف التي تسعى إلى تحقيقها. وبناء على ذلك و من أجل تحقيق أهداف الدراسة سيتم استخدام المنهج الاستقرائي والذي من خلاله يتم جمع البيانات والمعلومات من خلال الكتب والمراجع العربية والأجنبية ذات العلاقة بموضوع الدراسة، والدوريات والمقالات والتقارير، والأبحاث والدراسات السابقة المنشورة التي تتاولت موضوع الدارسة، ومن خلال البحث والمطالعة في مواقع الانترنت

خلصت الدراسة و من خلال مراجعة أدبيات الدراسات السابقة ذات العلاقة بموضوع الدراسة، إلى أن هنالك علاقة قوية بين أخلاقيات التسويق وولاء الزبون،وهذا ما أكدته دراسة (بن جروة، بن حوحو، مرجع سابق)، وكذلك تبين أن صدق الاعلان والرسائل الترويجية تؤثر على نظرة المستهلك الفلسطيني للأبعاد الأخلاقية للمسؤولية الاجتماعية للمؤسسات (حجاوي، مرجع سابق)، وأظهرت الدراسة الأثر السلبي الذي يتسبب به الاحتلال و الحصارعلى قطاع غزة، و كذلك الانقسام السياسي الفلسطيني، على الاقتصاد الفلسطيني والتتمية الاقتصادية في فلسطين وخاصة قطاع غزة، بما في ذلك قطاع الاتصالات موضوع الدراسة (SHAMMA، مرجع سابق)، (مرتجى، مرجع سابق)، لذلك توصي الدراسة شركتي جوال وأوريدو فلسطين الخلويتين بالاهتمام بأخلاقيات التسويق والعمل على كسب رضا الزبون في قطاع غزة، الذي يعاني من جراء احتلال ظالم وحصار شديد منذ عشرات السنوات، وهذا بدوره يعود بالفائدة على زيادة ربحية الثركتين، وكذلك على الخدمات المقدمة للمواطن في قطاع غزة.

المراجع

أبوحماد، محمود، 2011. التمويل الدولي للمؤسسات الأهلية الفلسطينية وأثثر على التتمية السياسية في قطاع غزة. (رسالة ماجستير) · جامعة الأزهر ، فلسطين. بالتل، التقرير السنوي، 1999.

بن جروة، حكيم، دلهوم، خليدة. 2015. "إمكانية تطبيق أبعاد تسويق العلاقات مع الزبون في الرفع من أداء المؤسسة التنافسي". المجلة الجزائرية للتمية الاقتصادية. العدد 02 . بودرجة، رمزي، صليحة، غلاب. 2017. "أثر التزام المؤسسات الاقتصادية بأخلاقيات التسويق على صورتها الذهنية". بحث مقدم للمؤتمر الدولي الثالث للاداء المتميز للمنظمات والحكومات، جامعة ورقلة، الجزائر. 
بن صالح، عبد الرزاق. 2014. أخلاقيات التسويق وأثرها على سلوك المستهلك. ( رسالة دكتوراة) جامعة الجزائر. توفيق، عبد الرحمن. 2007. علاقات العملاء. القاهرة:مركز الخبرات المهنية للإدارة. الطبعة الأولى. حجاوي، ياسمين. 2011. الأبعاد الأخلاقية التسويقية كما يدركها المستهلك الفلسطيني. (رسالة ماجستير). جامعة النجاح الفلسطينية.

خدير ، نسيمة. 2011. أخلاقيات الأعمال وتأثيرها على رضا الزبون. (رسالة ماجستير) . جامعة محمد بوقرة بومرداس. الدويك، أروى. 2006. مزيج الاتصالات التسويقي في شركات الهاتق الخلوي حالة تطبيقية على شركة جوال الفلسطينية. (رسلة ماجستير ). جامعة القدس.

سليمان، أمل. 2014. "أثر ولاء العميل على تحسين الميزة التتافسية لمصرف الجزيرة الأردني في السودان". مجلة

$$
\text { اماراباك. المجلد 05. العدد } 14 .
$$

سويدان، موسى. 2011. "تأثير الكلمة المنطوقة على قرار الشرائي للمستهلك". مجلة إدارة الأعمال المصرية. جامعة

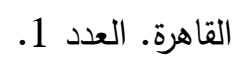

الطروانة، خالد. أبو جليل، محمد. 2013. أثر أخلاقيات الأعمال والمسؤولية الاجتماعية في تحقيق الميزة التتافسية - دراسة ميدانية في الثركات الصناعية المدرجة في سوق عمان المالي- بحث مقدم للمؤتمر الدولي الثاني لكلية إدارة الأعمال. جامعة مؤتة. الأردن. عبد العال، حازم. 2007. مدى تطبيق إدارة الجودة الثاملة في خدمة عملاء الهاتف الخلوي في شركة جوال من وجهة نظر العاملين بها في فلسطين. (رسالة ماجستير). الجامعة الاسلامية بغزة.

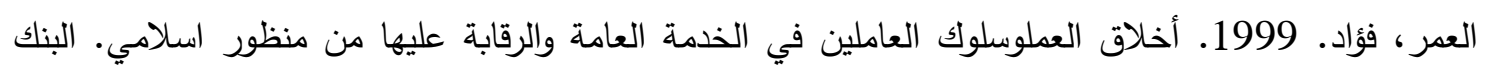

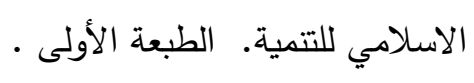

كافي، مصطفى. 2009. التسويق الاكتروني في ظل المتغيرات التكنولوجية المعاصرة. دمشق: دار ومؤسسة أرسلان للطباعة والنشر والتوزيع.

كامل، أميمة. 2006. "ثورة مرتقبة في مجال علاقة العملاء". مجلة الاتصالات والعالم الرقمي. العدد 146. كلي، لامية. 2016. أخلاقيات التسويق وتأثيرها على سلوك المستهلك النهائي. (رسالة ماجستير). جامعة أمجد بوقرة بومرداس.

مرتجى، زكي. 2014. "الانقسام الفلسطيني وأثره على التتمية بمحافظات غزة وسبل التغلب عليه". مجلة جامعة

$$
\text { القدس المفتوحة للأبحاث والدراسات. العدد } 34
$$

المرهضي، سنان. 2009. أثر نظام احوافز على أخلاقيات الوظيفة العامة دراسة ميدانية على عينة من الموظفين في المنظمات العامة. بحث أكاديمي. جامعة صنعاء. نجم، عبود. 2006. أخلاقيات الإدارة ومسؤولية الأعمال. عمان:مؤسسة الوراق للنشر والتوزيع. الهنداوي، ونس، وآخرون. 2010. "تقييم العلاقة بين تبني الثركات لأخلاقيات التسويق وفاعلية اعلانتها التجارية" • مجلة كلية بغداد للعلوم الاقتصادية الجامعية، العدد 25. 


$$
\text { يوسف، بومدين. 2015. 2018. }
$$

\section{References}

Al-Morhadi, S. (2009). The effect of the incentive system on the ethics of the public office, a field study on a sample of employees in public organizations. Sanaa' University. (in Arabic)

Abdelaal, H. (2007). The implantation of Total Quality Management in the services of cellular phone customers in Jawwal Company from the viewpoint of employees in Palestine. (Unpublished Master Thesis), The Islamic University, Gaza, Palestine. (in Arabic)

Abdulrazzaq, B. (2014). Marketing Ethics and Its Impact on Consumer Behavior." (Unpublished $\mathrm{PhD}$ Thesis), Algerian University. (in Arabic)

Abu Hammad, M. (2011). International funding for Palestinian civil institutions and its impact on political development in The Gaza Strip 2000-2010, (Unpublished Master Thesis), Al-Azhar University, Gaza, Palestine. (in Arabic)

Alareeni, B. (2018). Does corporate governance influence earnings management in listed companies in Bahrain Bourse?, Journal of Asia Business Studies, 2(4), 551-570. https://doi.org/10.1108/JABS-06-2017-0082

Alareeni, B., \& Branson, J. (2013). Predicting Listed Companies' Failure in Jordan Using Altman Models: A Case Study. International Journal of Business and Management, 8(1), 113-126. https://doi.org/10.5539/ijbm.v8n1p113

Alareeni, B.A. (2019). The associations between audit firm attributes and audit qualityspecific indicators: A meta-analysis, Managerial Auditing Journal, 34(1), 6-43. https://doi.org/10.1108/MAJ-05-2017-1559

Al-Hindawi, W., Hasona, A. and Foraihat, S. (2010). Evaluating the relationship between companies' adoption of marketing ethics and the effectiveness of their commercial advertisements: A study of Jordanian cellular telecommunications companies. Journal of Baghdad College of Economic Sciences University. 25, 69-90. (in Arabic)

Al-Omar, F. (1999). Work ethics and the behavior of employees in public service and control over it from an Islamic perspective. 1st edition. The Islamic Development Bank. (in Arabic)

Alqallaf, H. and Alareeni, B. (2018). Evolving of Selected Integrated Reporting Capitals among Listed Bahraini Banks, International Journal of Business Ethics and Governance, 1(1), 15-36. https://doi.org/10.51325/ijbeg.v1i1.10

Altarawna, N. and Abu-Jalil, M. (2013). The effect of business ethics and social responsibilty in obtaining Competitive advantage-field study in industrial companies listed in the Amman Stock Exchange.The International Conference of the College of Business Administration. Mutah University. Jordan. (in Arabic).

Bin Jarwa, H. and Delhoum, K. (2015). The possibility of applying marketing relationships with the customer in the dimensions of lifting of the competitive enterprise performance. Algerian Journal for Economic Development. 2(1), 91-104. (in Arabic) https://doi.org/10.12816/0020030 
Bodarja, R. and Ghallab S. (2017). The Impact of Economic Institutions on Marketing Ethics on Their Mental Image in the 3rd international conference on the distinguished performance of organizations and governments. (in Arabic)

Chen, Y. C., Shen, Y. C., \& Liao, S. (2009). An integrated model of customer loyalty: an empirical examination in retailing practice. The Service Industries Journal, 29(3), 267-280. https://doi.org/10.1080/02642060701842886

Dweik, A. (2006). The combination of marketing communications in the cellular phone companies Application status 'Jawwal Palestinian', (Unpublished Master Thesis), Al-Quds University, Palestine. (in Arabic)

Fournier, S., \& Yao, J. L. (1997). Reviving brand loyalty: A reconceptualization within the framework of consumer-brand relationships. International Journal of research in Marketing, 14(5), 451-472. https://doi.org/10.1016/S0167-8116(97)00021-9

Fülöp, G., Hisrich, R. D., \& Szegedi, K. (2000). Business ethics and social responsibility in transition economies. Journal of Management Development, 19(1). https://doi.org/10.1108/02621710010308135

Furlong, C. B. (1993). Marketing for keeps: building your business by retaining your customers. John Wiley \& Sons Inc.

Hijjawi, Y. (2011). Marketing ethical dimensions as perceived by the Palestinian consumer, (Unpublished Master Thesis), Alnajah University, Palestine. (in Arabic)

Joel, W. (2001). Customer Retention of Business Travelers in the Airline Industry. Unpublished dissertation, United States International University, San Diego.

Kafi, M. (2009). E-marketing considering recent changes. 1st Edition. Dar Ruslan for Printing, Publishing and Distribution. Damascus. (in Arabic)

Kahli, L. (2016). Marketing Ethics and Its Impact on Final Consumer Behavior: A Case Study of National Food Products Consumers in Boumerdes State. (Unpublished Master Thesis), Université M'hamed Bougara Boumerdes, Algeria. (in Arabic)

Kamel, O. (2006). An upcoming revolution in customer relationship. Telecom \& Digital World Magazine. 146. (in Arabic)

Khdair, N. (2011). Business ethics and its impact on customer satisfaction, (Unpublished Master Thesis), Université M'hamed Bougara Boumerdes, Algeria. (in Arabic)

Murtaja, Z. (2014). Palestinian Fragmentation and its Impact on Development in Gaza Governorates and Means of Overcoming it. Journal of Al-Quds open University for Research and Studies, 34(1), 267-306. (in Arabic)

Najem, A. (2006). Management ethics and business responsibility. Al-Waraq for Publication and Distribution. Amman, Jordan. (in Arabic)

Palestinian Telecommunications company (Paltel), (1999). Annual Report. Retrieved from: www.paltel.ps. (in Arabic)

Shamma, A. (1986). Children in war, world health association, july, 6-13.

Suliman, A. (2014). The impact of client loyalty on the improve of competitive advantage to Jordanian Gazeera Bank in Sudan. Amarabac Magazin. 5(14), 95-108. (in Arabic)

Sweidan, N. (2011). The Effect of the Word-of Mouth on the Consumer Purchasing Decision. Name Egyptian Journal of Business Administration. 1, Cairo University. (in Arabic)

Tawfiq, A. (2007). Customer Relationship Management. 1st edition. Professional Management Expertise Center. Ciro. (in Arabic)

Yousif, B. (2015). Moral Works and Governance's Practices in Contemporary Works Organizations. Journal of Economics and Finance, 1(1), 76-96. (in Arabic) 